\title{
In Vitro Examination of the Safety of Rotational Atherectomy of Side Branches Jailed by Stents
}

\author{
Hirotaka Oda, MD; Tsutomu Miida, MD; Tetsurou Toeda, MD; \\ Norio Higuma, MD; Kazuyoshi Takahashi, MD
}

\begin{abstract}
In vitro experimental models of branch orifices jailed by various stents were created to estimate the safety and the efficacy of rotational atherectomy when rotational burrs were advanced through the struts of stents. The scaffolding structures of the stents were destroyed due to loss and deflection of the struts, and the size of ablated stent-particles differed: the maximal size was $1.7 \mathrm{~mm}$ in slotted stents, and $17.6 \mathrm{~mm}$ in coiled stents. Thus, there is a definite potential for ablating stents when rotational atherectomy of restenotic lesions of side-branch orifices jailed by stents is performed. (Jpn Circ J 1999; 63: 537-541)
\end{abstract}

Key Words: Rotational coronary atherectomy; Side branch; Stent jail; Wire bias

$\mathbf{S}$ ome reports have shown that angioplasty using lowprofile balloons was successful in reopening orifices of branches jailed by stents ${ }^{1}$ and that coronary stenting into side branches could be performed through the struts of stents that had been implanted in the trunk vessels 2,3 There have been recent reports of rotational atherectomy used to successfully ablate stenosis of branches compromised by stents4 Yet, when rotational burrs are passed through stent struts, these might destroy the structure of the implanted stent, failing to ablate the ostial lesions of the branches satisfactorily. Thus, it is not known whether the use of rotational atherectomy is safe and effective. This report describes an in vitro examination to estimate the safety and efficacy of rotational atherectomy when rotational burrs are advanced through the stent struts.

\section{Methods \\ Experiment Description \\ We created in vitro experimental models of branch orifices jailed by stents. A 2.0 -mm diameter hole represent- ing a stenotic ostium of a side branch was drilled into a silicon tube with an inner diameter of $3.0 \mathrm{~mm}$ and a wall thickness of $1.0 \mathrm{~mm}$. In the first step, stents of $3.5-\mathrm{mm}$ diameter were implanted at the holes of the tubes and balloons with a 3.5-mm diameter (High Energy ${ }^{\mathrm{TM}}$, Boston Scientific Co, Natick, MA, USA) were inflated to a post- dilatory pressure of 15 ATMs (Fig 1A). In the second step, 0.014-inch guide wires were passed through the stent struts and through the holes representing the ostial stenotic side branches, and the relapped used balloons with a $3.0-\mathrm{mm}$ diameter (High Energy ${ }^{\mathrm{TM}}$ ) were advanced through the holes and inflated to 12 ATMs (Fig 1B). In the third step, the 0.014-inch guide wires in the biggest intra-strut spaces were exchanged with 0.010 -inch guide wires and the holes were ablated by $1.75-\mathrm{mm}$ burrs of Rotablator ${ }^{\mathrm{TM}}$ (Boston Scientific Co) with a rotational speed of $180,000 \mathrm{rpm}$. The}

(Received January 17, 1999; accepted April 9, 1999)

Department of Cardiology, Niigata City General Hospital, Niigata, Japan

Mailing address: Hirotaka Oda, MD, The Department of Cardiology, Niigata City General Hospital, Shichikuyama 2-6-1, Niigata 9508739, Japan
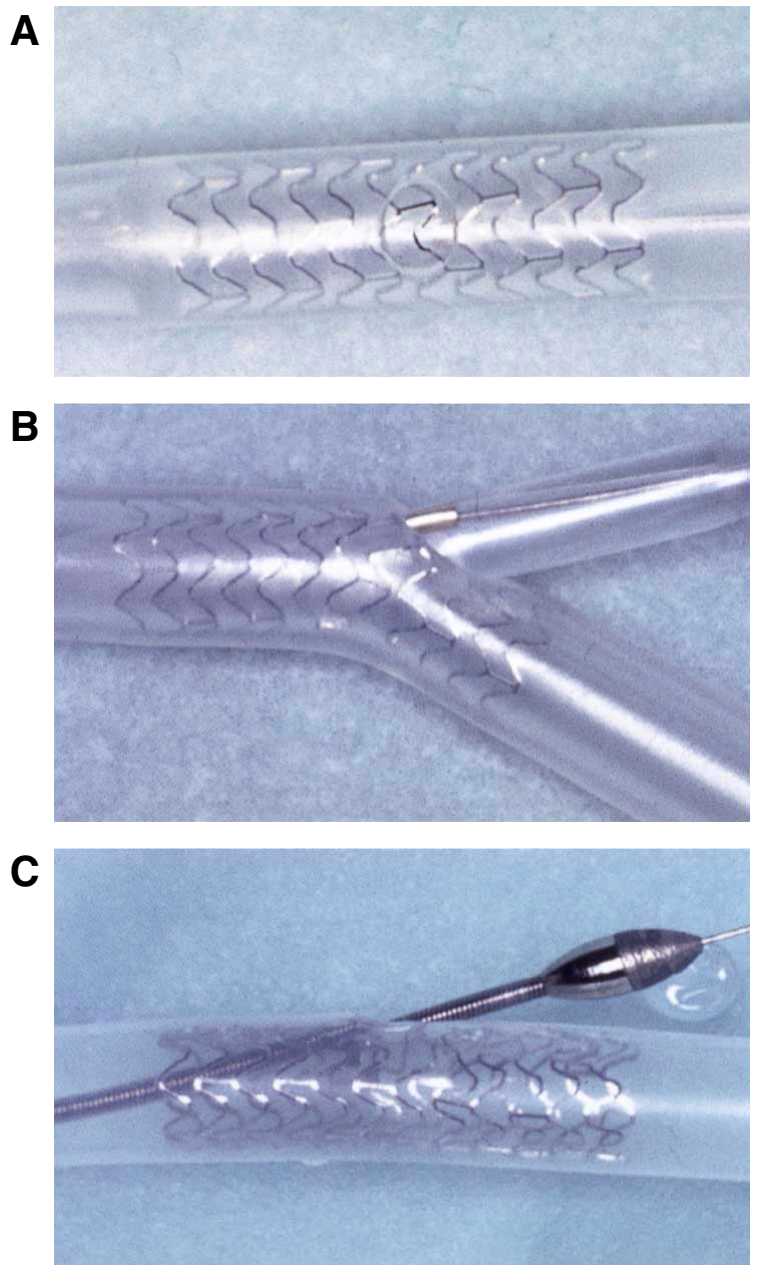

Fig 1. In vitro examination of rotational atherectomy of side branches jailed by stents. (A) First, a Multi-Link ${ }^{\mathrm{TM}}$ stent with a diameter of $3.5 \mathrm{~mm}$ was implanted at a hole of a tube and a balloon with a diameter of $3.5 \mathrm{~mm}$ was postdilatorily inflated to 15 ATMs. (B) Second, a 0.014-inch guide wire was passed through the stent strut and through the hole, and a balloon with a diameter of $3.0 \mathrm{~mm}$ was advanced into the hole and inflated to 12 ATMs. (C) Third, the 0.014inch guide wire in the biggest intra-strut space was exchanged with a 0.010 -inch guide wire, and the hole was ablated with a $1.75 \mathrm{~mm}$-burr. 
A

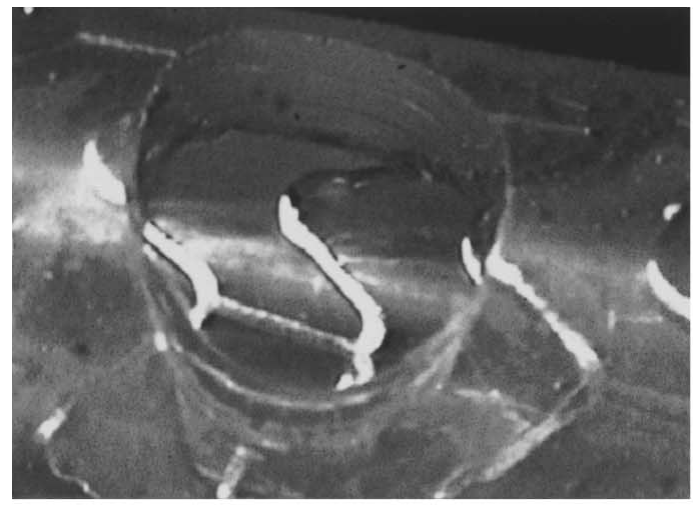

C

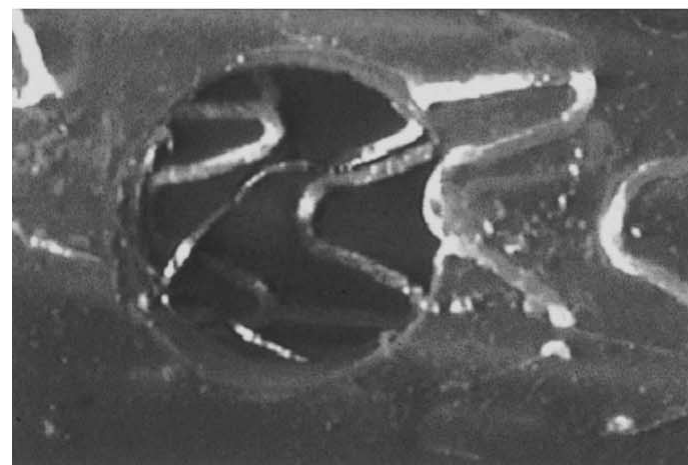

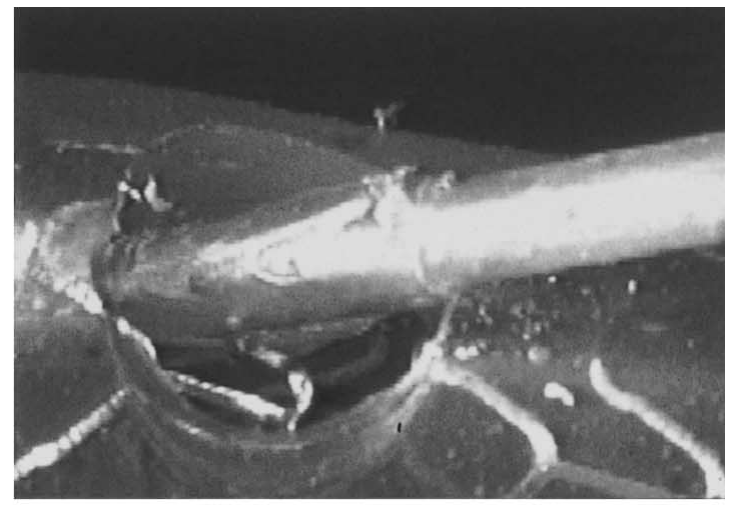

B

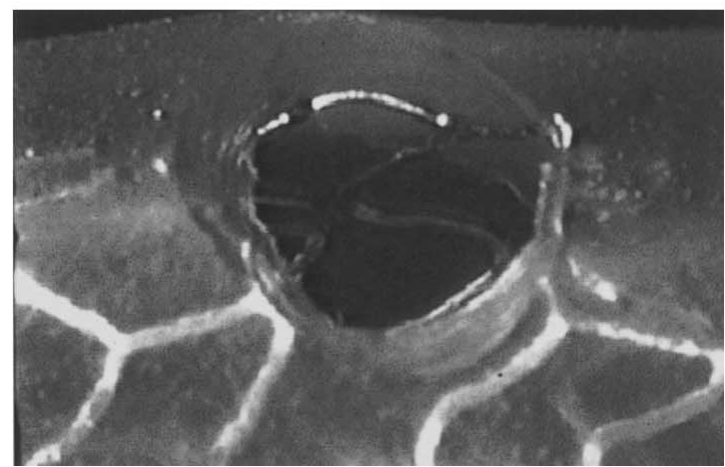

Fig 2. Angioplasty of intra-strut spaces of a Multi-Link ${ }^{\mathrm{TM}}$ stent. (A) A Multi-Link ${ }^{\mathrm{TM}}$ stent with a diameter of $3.5 \mathrm{~mm}$ was implanted at a side hole within a silicon tube and a balloon with a diameter of $3.5 \mathrm{~mm}$ was postdiatorily inflated to 15 ATMs. (B) A 3.0 -mm balloon was placed into the side hole through the stent, deflecting a strut into the main lumen. (C) Following side branch dilatation with 3.0-mm balloon, a strut was seen to further protrude into the silicon tube lumen. (D) When the 3.0-mm balloon pushed the strut out of the hole during the balloon advancement, a space of struts with a short diameter of about $3.0 \mathrm{~mm}$ was gotten after side branch dilatation whereas struts did not protrude into the main lumen.

A

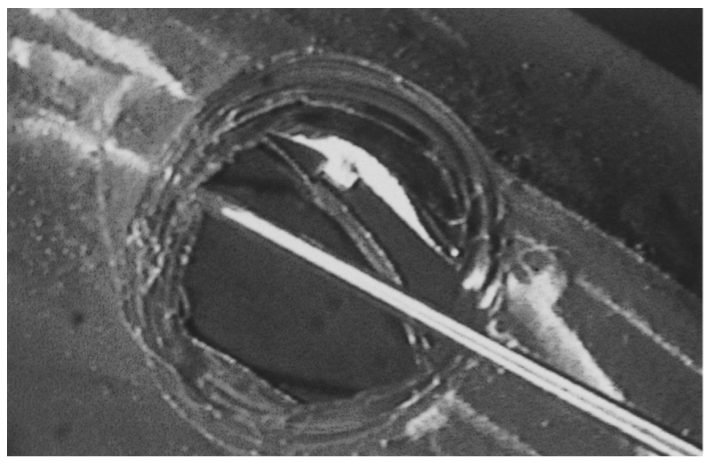

C

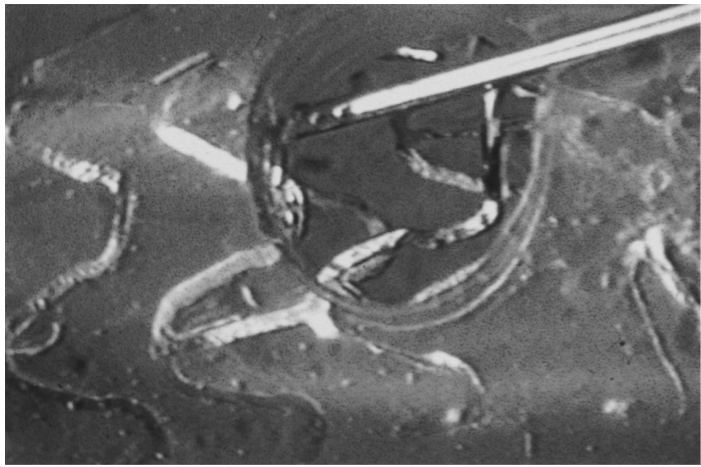

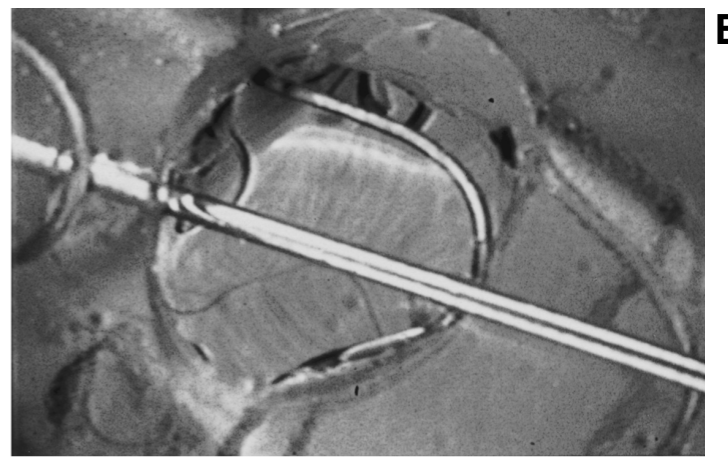

B

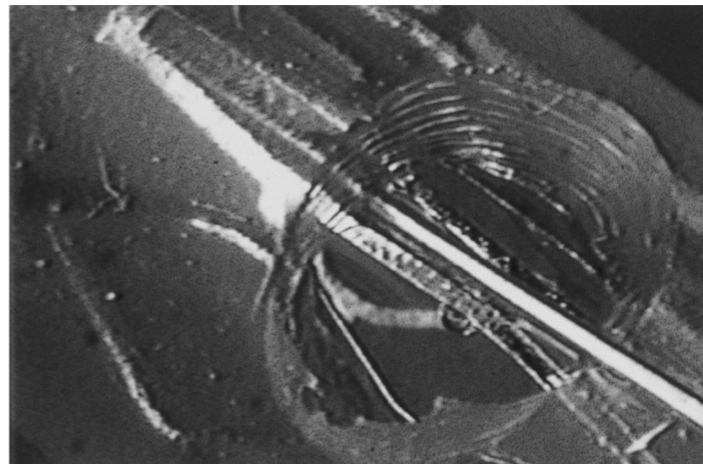

Fig 3. In the third step, 0.010-inch guide wires were passed through the intra-strut spaces and through the holes. (A) Spiral PalmazSchatz ${ }^{\mathrm{TM}}$ stent for previous angioplasty of intra-strut spaces, (B) Wiktor ${ }^{\mathrm{TM}}$ stent for previous angioplasty of intra-strut spaces, (C) Multi-Link $^{\mathrm{TM}}$ stent for previous angioplasty of intra-strut spaces, (D) A standard Palmaz-Schatz ${ }^{\mathrm{TM}}$ stent, which did not undergo side branch dilatation. 
A
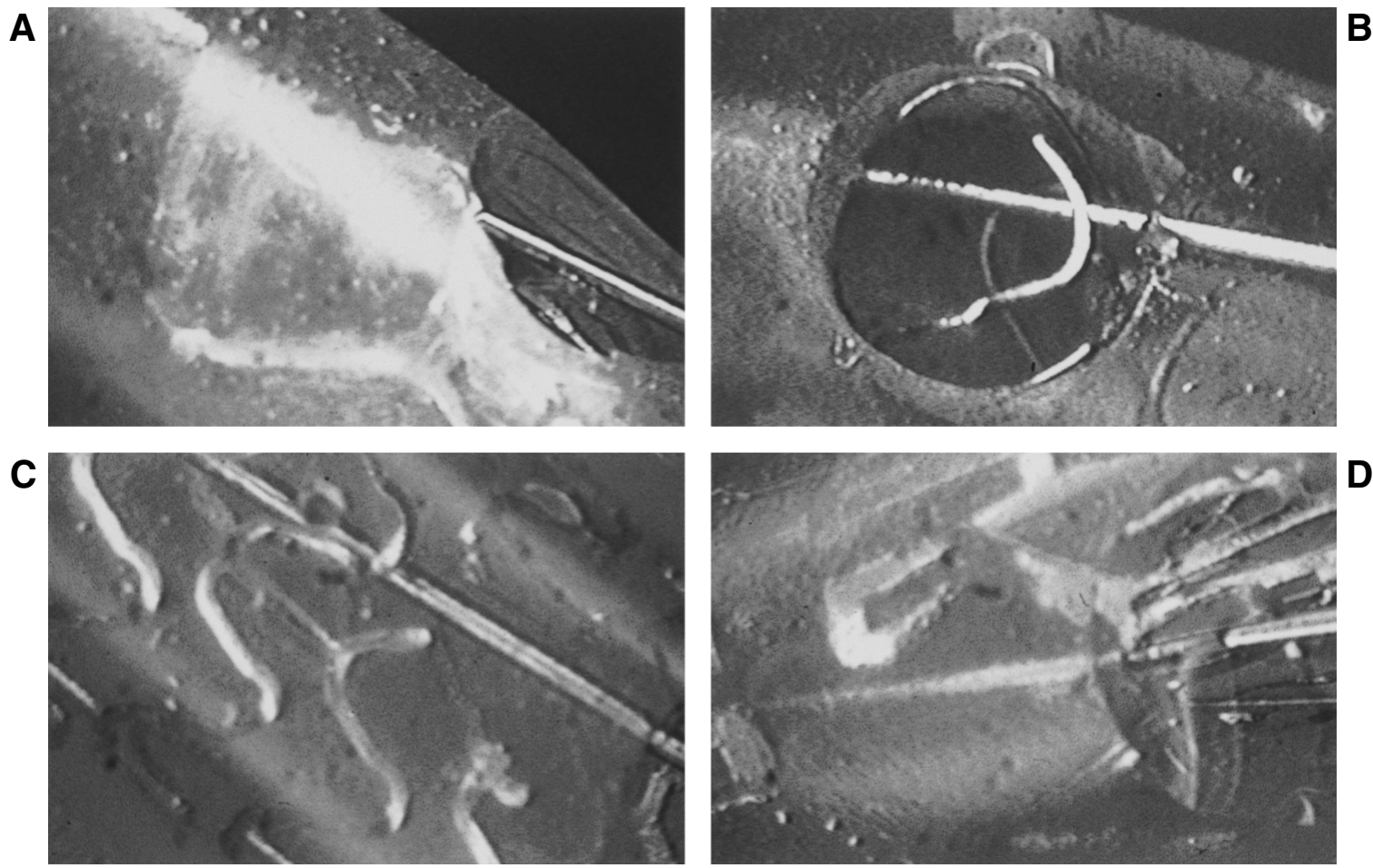

Fig 4. After rotational atherectomy. (A) Spiral Palmaz-Schatz ${ }^{\mathrm{TM}}$ stent for previous angioplasty of intra-strut spaces, (B) Wiktor ${ }^{\mathrm{TM}}$ stent for previous angioplasty of intra-strut spaces, (C) Multi-Link ${ }^{\mathrm{TM}}$ stent for previous angioplasty of intra-strut spaces, (D) A standard Palmaz-Schatz ${ }^{\mathrm{TM}}$ stent without prior angioplasty of intra-strut spaces.

A
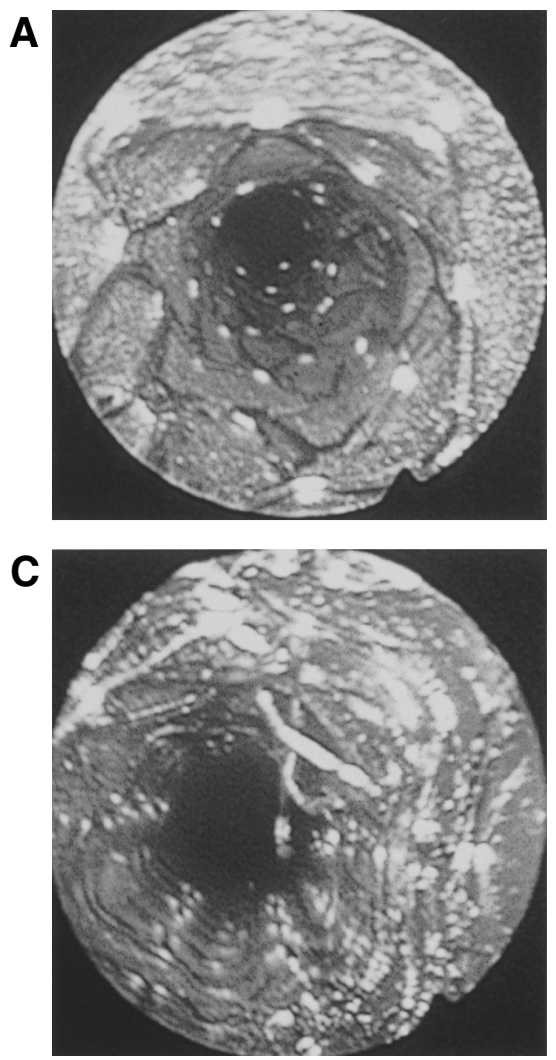
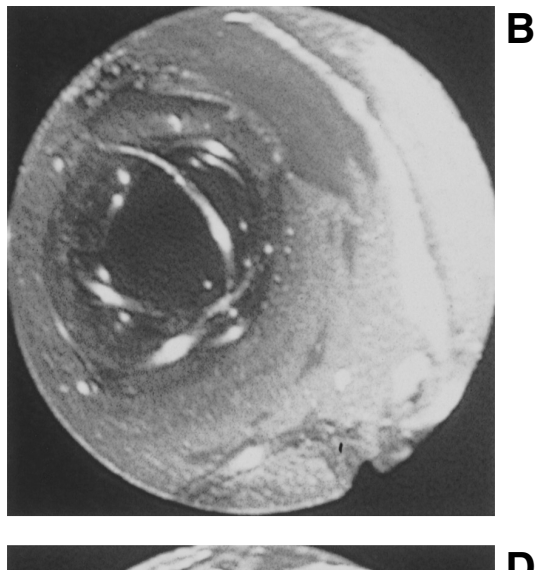

B

D

Fig 5. Self-scope findings after rotational atherectomy. (A) Spiral Palmaz-Schatz ${ }^{\mathrm{TM}}$ stent for angioplasty of intra-strut spaces, (B) Wiktor ${ }^{\mathrm{TM}}$ stent for angioplasty of intra-strut spaces, (C) Multi-Link ${ }^{\mathrm{TM}}$ stent for angioplasty of intra-strut spaces, (D) A standard PalmazSchatz ${ }^{\mathrm{TM}}$ stent without prior angioplasty of intra-strut spaces.

rotational burrs were made to traverse the hole in all stents (Fig 1C). A self-scope system (A7005, Olympus, Tokyo, Japan) and a video macroscope system (VMS-1300, Nihon
Kohden, Tokyo, Japan) were used for close observation of the stent's structures. For these experiments, we used Palmaz-Schatz ${ }^{\mathrm{TM}}$ stents of the standard type and spiral type 


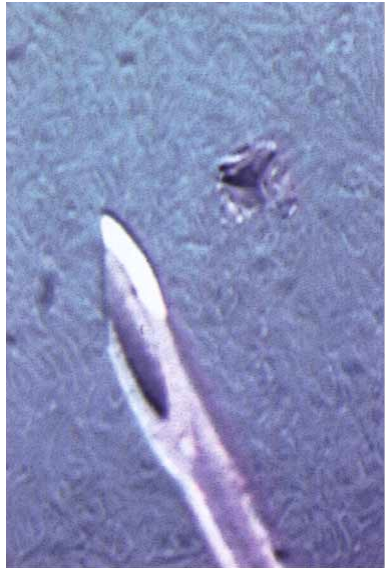

A

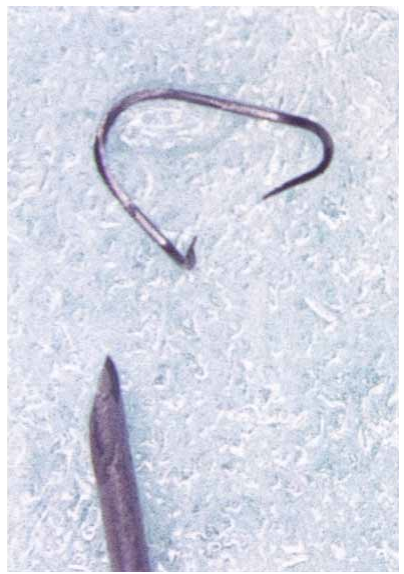

B
Fig 6. Ablated particles. (A) A standard Palmaz-Schatz ${ }^{\mathrm{TM}}$ stent, (B) Wiktor ${ }^{\mathrm{TM}}$ stent. The needles are 18 gauge.

with lengths of $15 \mathrm{~mm}$ and $14 \mathrm{~mm}$, respectively (Johnson \& Johnson Interventional Systems Co, Warren, NJ, USA), Wiktor ${ }^{\mathrm{TM}}$ stents with lengths of $16 \mathrm{~mm}$ (Medtronic, Inc, Minneapolis, MN, USA), and Multi-Link ${ }^{\mathrm{TM}}$ stents with lengths of $15 \mathrm{~mm}$ (Advanced Cardiovascular System, Inc, Temecula, CA, USA). A 3.5-mm standard PalmazSchatz $^{\mathrm{TM}}$ stent was implanted to span the hole, while the proximal of the 2 tubular slotted halves was placed under the hole. Standard Palmaz-Schatz stents were divided into 2 groups: in group 1 all 3 steps were performed, whereas for group 2, step 2 of the 3 procedures was omitted. These 3 steps were repeated twice for all kinds of stents.

In the second step, using Palmaz-Schatz ${ }^{\mathrm{TM}}$ stents of the standard type and spiral type, the $3.0-\mathrm{mm}$ balloons were not fully dilated in the strut positions, so that the balloon looked like an hour glass. Moreover, the diamond-shaped space in the strut changed to an ellipse-shaped space with a short diameter of about $2.3 \mathrm{~mm}$ after balloon inflation? For Wiktor $^{\mathrm{TM}}$ and Multi-Link ${ }^{\mathrm{TM}}$ stents the $3.0-\mathrm{mm}$ balloons were fully dilated (Fig 1B). Yet when the balloons were advanced through the holes in the Multi-Link ${ }^{\mathrm{TM}}$ stent, if the balloon rode on the strut under the hole and pushed the strut into the lumen (Fig 2B), the strut protruded inside the silicon tube lumen after balloon inflation(Fig 2C). If the balloon pushed the strut out of the hole, a space of struts with a short diameter of about $3.0 \mathrm{~mm}$ appeared under the hole after balloon inflation (Fig 2D). For the Multi-Link ${ }^{\mathrm{TM}}$ stents the latter models were used. Moreover, the selfscope findings at this second step revealed none of the stents protruding struts inside the lumen. In the third step, 0.010-inch guide wires were passed through the biggest intra-strut spaces and through the holes (Fig 3A-C). However, with the standard Palmaz-Schatz ${ }^{\mathrm{TM}}$ stents, angioplasty was not performed in the hole, and the 0.010-inch guide wires were located in the narrow intra-strut space (Fig 3D). All wires were directed to the side of the holes because of the bifurcational curves. We ablated the holes with $1.75-\mathrm{mm}$ burrs with a rotational speed of $180,000 \mathrm{rpm}$, and attempted to run the burrs without a drop in rpm of more than 5,000 while avoiding aggressive burr advancement. However, we sometimes experienced decreases of more than $10,000 \mathrm{rpm}$ in all stents. The rotational burrs were made to traverse the hole in all stents.

In slotted stents such as the Palmaz-Schatz ${ }^{\mathrm{TM}}$ and MultiLink $^{\mathrm{TM}}$ stents, struts and tube walls, which were located on the near sides of the wires and the holes, were ablated. The scaffolding structures of the stents were destroyed due to loss and deflection of struts (Fig 4A, C, D). The self-scope findings revealed that Multi-Link ${ }^{\mathrm{TM}}$ stents had struts protruding into the lumen whereas the Palmaz-Schatz ${ }^{\mathrm{TM}}$ stents apparently had no protruding struts (Fig 5A, C, D). The size of ablated particles differed: the maximal size was $1.7 \mathrm{~mm}$ (Fig 6A). For Wiktor ${ }^{\mathrm{TM}}$ stents, a strut with a length of $17.6 \mathrm{~mm}$ was cut out with extremely sharp edges (Fig 6B), deflecting the struts near the hole (Fig 4B). The self-scope findings showed protruding struts inside the lumen (Fig 5C).

\section{Discussion}

In this study, we used 2 types of stents: coiled and slotted. For coiled Wiktor ${ }^{\mathrm{TM}}$ stents, the size of the strut cutoff was larger than expected and it certainly seemed to anchor in the distal artery wall. Moreover, the edges of the ablated struts were extremely sharp and protruded into the lumen. Thus, when postdilator balloon angioplasty is performed, balloon rupture occurs. With slotted PalmazSchatz $^{\mathrm{TM}}$ or Multi-Link ${ }^{\mathrm{TM}}$ stents, if balloon angioplasty using $3.0-\mathrm{mm}$ balloons is performed within the intra-strut spaces before rotational ablation, the minimal diameters of the intra-strut spaces will be about 2.3 or $3.0 \mathrm{~mm}$, which is larger than the $1.75 \mathrm{~mm}$ of the burr size. Dauerman et al reported that it was important that angioplasty had been performed in the intra-strut spaces during the initial intervention and that burr sizes should be less than the minimal diameter of intra-strut spaces when rotational atherectomy was used for restenotic branches jailed by stent. However, we must remember the 'wire bias' of guide wires; when a wire is placed in an angulated lesion, the wire is located in the inner curve of the lesion and the rotational ablation is directed to the inner curve by the guide wire? During rotational atherectomy of the branch orifices jailed by stent the wires are directed to the side of the walls from which the branches arise. Thus, the stent struts of this side will be ablated as shown in Fig 4A,C. Particle size differs: the maximal size was $1.7 \mathrm{~mm}$. Particles of this size will cause distal embolization. For slotted stents within which branch vessel balloon angioplasty is not performed in the intrastrut spaces before rotational ablation, rotational atherectomy will destroy many parts of the stent. Therefore, the volume of embolizing metal parts seems to be greater when compared with cases in which balloon angioplasty is performed before rotational ablation. However, in the present study, we did not estimate the size and the volume of ablated particles in detail. The extent of potential myocardial damage from embolization of particles is unknown, because it has not yet been tested in in-vivo experiments.

\section{Study Limitation}

The stents used in the present study are different from stents which the endothelium has covered several weeks and months after stent implantation; the effects of rotational atherctomy may be different between the 2 because the thick endothelium will prevent rotational burrs from ablating struts covered by the endothelium. However, this new endothelium is soft. Thus, there is possibility that burrs will touch and ablate stent-struts after easy ablation of the soft endothelium covering the stents, and the effect of 'wire bias' 5 will play an important part in ablating struts. 
Based on the results of our in vitro examinations, there is definite potential for ablating stents when rotational atherectomy of restenotic lesions of side-branch orifices jailed by stents is performed.

\section{Acknowledgments}

The authors thank Ogi Medical Science Co. for technical assistance and Gerard P. Grillo for assistance.

\section{References}

1. Caputo RP, Chafizadeh ER, Stoler RC, Lopez JJ, Cohen DJ, Kuntz
RE, et al: Stent jail: A minimum-security prison. Am J Cardiol 1996; 77: $1226-1230$

2. Nakamura S, Hall P, Maiellio L, Colombo A: Techniques for Palmaz-Schatz stent deployment in lesions with a large side branch. Cathet Cardiovasc Diagn 1995; 34: 353-361

3. Oda H, Ito E, Miida T, Toeda T, Higuma N: 'Fork' stenting for bifurcational lesion. J Interven Cardiol 1996; 9: 445-454

4. Dauerman HL, Cohen DJ, Carrozza JP, Kuntz RE, Baim DS : Rotational atherectomy for the treatment of restenotic ostial side branches jailed by prior stent placement. Cathet Cardiovasc Diagn 1998; 43: 447-450

5. Reisman M, Harms V: Guidewire bias: Potential source of complications with rotational atherectomy. Cathet Cardiovasc Diagn 1994; Suppl 3: 64-68 